\title{
Optical projects in the Clinic program at Harvey Mudd College
}

\section{Q. Yang}

Q. Yang, "Optical projects in the Clinic program at Harvey Mudd College," Proc. SPIE 10452, 14th Conference on Education and Training in Optics and Photonics: ETOP 2017, 104520D (16 August 2017); doi: 10.1117/12.2266334

SDIE Event: 14th Conference on Education and Training in Optics and Photonics, ETOP 2017, 2017, Hangzhou, China 


\title{
Optical Projects in the Clinic Program at Harvey Mudd College
}

\author{
Q. Yang \\ Engineering Department, Harvey Mudd College, Claremont, CA 91711
}

\begin{abstract}
Clinic program is the senior capstone program at Harvey Mudd College (HMC). Multidisciplinary and industry-sponsored projects allow a team of students to solve a real-world problem over one academic year. Over its 50 plus years, Clinic program has completed numerous optics related projects. This report gives an overview of the Clinic program, reviews recent optical projects and discusses how this program supports the learning of the HMC engineering students. A few sample optical projects with more details are presented to provide an insight of what challenges that undergraduates can overcome. Students achieve learning within the optics discipline and the related engineering disciplines. The experiences in these optical projects indicate the great potential to bringing optical hands-on projects into the undergraduate level. Because of the general engineering curriculum at HMC, these projects often work the best with a multidisciplinary nature even if the core of the project is optically focused. Students gain leadership training, oral and written communication skills and experiences in team work. Close relationship with the sponsor liaisons allows for the students to gain skills in professional conduct, management of tight schedule and a specified budget, and it well prepares the students to their engineering practice. Optical projects have their own sets of specific challenges, so it needs to be chosen properly to match the undergraduate skill sets such as those of HMC engineering students.
\end{abstract}

Keywords: capstone project, Harvey Mudd College, HMC, Clinic, undergraduate education, hands-on experience, optics, fiber, LED, multidisciplinary

\section{INTRODUCTION}

The Clinic program, an extraordinary program of collaboration between industry and Harvey Mudd College that has been a hallmark of this institution for more than 50 years, engages juniors and seniors in the solution of real-world, technical problems for industrial clients. [1] The term "Clinic" borrows from medical practice when new doctors are taught to practice their skills with real patients in hospitals in their last stage of academic training. They work directly on the real medical problems and cases, and learn from the more experienced doctors and gain practice skills. The vision of the Clinic program is that engineers in training ought to work on real-world engineering problems like doctors before graduation. By working directly on an open-ended problem (without a unique solution), and working closely with the industry liaisons, students gain tremendous practice and professional training through these projects. Since the real world is full of problems waiting for engineering solutions, the effort goes into the Clinic program is not only for the education of the students, which is the core value of the Clinic for the college and for the students, but also for its delivered solution and outcome to impact the real industry as a byproduct. Currently in HMC, Clinic program runs across multiple departments, including Engineering, Computer Science, Physics and Mathematics department. There are also a small number of Global Clinic projects when an oversea industry sponsor and often an oversea partner university are jointly involved in such a project.

All Clinic projects are recruited from the industry sponsor, and each project allows for a team of 4 to 6 students to solve a real-world problem over one academic year. Since Clinic is a required course in Engineering department, one year for the senior students and one semester for the junior students, the size of the program or the number of engineering Clinic projects fluctuates slightly from year to year based on the number of major students. An engineering Clinic team is mixed of seniors and juniors and juniors switch to new team members between the semesters to simulate the transition of team personal as that happens in the industry setting. One (sometimes two) faculty advisor is assigned to each project. A lot of effort goes behind the scene to establish the portfolio of projects with a wide variety of choices of disciplines, which might be one of the operating challenges of the program. Sponsors commit a fixed fee and internally we have certain structures to support the financial needs of the overall program. But in this report, we primarily focus our discussion on the educational benefit of the program and how it impacts the student learning. It is quite often to have a joint project across two departments (Engineering, Computer Science, Physics and Mathematics) due to the multidisciplinary nature of many

14th Conference on Education and Training in Optics and Photonics: ETOP 2017, edited by Xu Liu,

Xi-Cheng Zhang, Proc. of SPIE Vol. 10452, 104520D · C 2017 ICO, IEEE, OSA, SPIE

CCC code: $0277-786 \mathrm{X} / 17 / \$ 18 \cdot$ doi: $10.1117 / 12.2266334$

Proc. of SPIE Vol. 10452 104520D-1 
projects. Because Clinic is established as a college program, there are many benefits of one supporting structure across different departments to run the program operation throughout the year. This involves complicated contracting stage and financial management with a variety of sponsor entities (companies, educational institutions, national labs and non-profit organizations). In addition, many common events on campus such as early meet between students and sponsors and final projects day that wraps up the projects should be carefully coordinated. In the first semester, the teams conduct design review sessions with several other teams in less formal presentations, where the students solicit input and feedback from their peers and faculty. In the second semester, more formal presentations are delivered so that students get to learn about other projects and share their own work in a professional fashion. Liaisons are also invited to the spring semester presentations. Additional site visits and face-to-face meeting are often arranged to facilitate the project progress. Students and the faculty advisor individually manage their own team progress by working closely with the technical liaisons from their sponsor. Regular weekly feedback is critically important to track the progress of the project. When facing real problems, important skills such as communication, team work and leadership can be flexibly and naturally incorporated as the project proceeds. A budget is requested at the beginning of the semester by each team and approved by the Clinic directors who oversee the recruitment of the projects as well as higher level Clinic program management. The overall program allows for a broader educational experience than more specific optical engineering specialties, however, the exposure to various technical aspects of the optical project brings great benefits, opportunities and challenges. These projects provide a great match with the broader educational goal of the general engineering program at HMC. While the Clinic program is not specifically designed for optical engineering education, a variety of optical projects have been completed throughout the years. In section 2, a good sample of these past optical projects will be discussed with more specific details. In section 3 and 4, the report will review and summarize some of the important undergraduate skills as learning outcome of the program, how learning optics happens in the Clinic program and some of the challenges that we observed. My personal experiences as a faculty advisor will also be shared throughout. We hope such experiences and lessons provide values for other institutions and educators who consider to incorporate hands-on experience and often multidisciplinary nature of the optical engineering in the undergraduate level.

\section{SAMPLE OPTICAL PROJECTS}

In this section, we provide three different optical project samples over a long span of time. While the specific technology involved in each project has evolved quite a bit in recent years, in the educational context, it is less relevant in terms of training undergraduates' practical skills. A brief project statement and background will be provided and the final deliverables of the team will also be provided. We will review some of the main challenges that the students face in these projects. The outcome in each project provides a good sample and insight in terms of overall success of the projects and effectiveness of such hands-on educational efforts. We will review experiences that best assist students in researching the relevant background technology and efficiently applying their knowledge in such open-ended problems with limited time and financial resources. These sample projects provide a good variety of scopes and applications for the optical technologies in an institution with broad engineering educational emphasis. Specifically, the four projects are (1) Federal Aviation Administration, "Update and Integration of LED Airport Approach Lighting" in 2002-2003, (2) Ultra Violet Product Inc. "Uniform Illumination for Fluorescent In Vivo Imaging" in 2005-2006, (3) City of Hope, "Redesigning the Lighting and Optics for Laparoscopic Surgery", 2016-2017. From these experiences, we review a set of common potential areas for bringing in optical projects in the undergraduate level, specifically for a program such as HMC Clinic that is not targeted at optical engineering education.

\subsection{Project 1: Update and Integration of LED Airport Approach Lighting- FAA}

Airport runways use incandescent approaching light systems. This FAA project is an early exploration of replacing the current lighting systems with LED and maintaining comparable luminescence. The team built prototypes for two different light approaching systems (MALSR and ALSF-2 system respectively). The main body of work includes LED selections, electrical power management, luminance measurement, LED configuration and light bar mechanical design, and field testing. Some specific challenges are highlighted below:

1. High power LEDs for such an application were not commercially abundant in year 2002-2003. As a result, the team chose Luxeon Star LEDs with a rather limited number of options in terms of power and specifications.

2. There are some power interface challenges between what's available in the airport and the LED devices. Between MALSR (Medium Intensity Approaching Lighting System) and ALSF-2 (High Intensity Approaching System), there are different standards involved. For example, MALSR is a voltage-based system, and the existing MALSR 
system steps down from 120VAC to 50VAC for the incandescent bulbs. While ALSF-2 system is a current driving system, and it uses power regular to support 5 different AC current levels for the different operational needs. LED devices are DC current driven, so the circuit interface between existing power structure for the two systems and the LED light bar prototypes must be resolved. All the design alternatives must meet the FAA regulation, and some modifications are not possible with the existing airport power and cabling standards. A simple bridge rectifier and filter system was chosen to convert the AC voltage to DC voltage. Resistor networks were incorporated carefully to control the required luminance based on the theoretical analysis and estimates. For the ALSF-2 system, the incoming current level was much higher than what's required and handled by LEDs ( $\sim 30$ times more), therefore, current transformers were incorporated to step down the current to each string of LEDs. Rectifier and filter circuit was similarly incorporated to convert from the initial AC circuit to DC circuit.

3. The design of the light bar must incorporate reliability consideration. The team decided on 5 parallel groups, each with 16 LEDs in series to make up the sufficient luminance and power. The choice of the current regulating resistor must be able to handle large amount of the power because these LEDs are running at high power level for sufficient luminance. The light bar built from aluminum served well as the structural support and provided the heat sink needs.

4. Testing was completed both in the lab as well as the field. In the lab, we can test the power consumption of the whole bar and reliability of the overall system. Field testing is extremely important because LED light color is different from incandescent lighting. Even though luminance is one way of measurement, it is ultimately to the eyes of the pilots who uses the flight approaching system during the landing process. The optics of LED is not optimally designed for such an application; therefore, a luminance estimate for short distance is quite different from above sky. This can only be examined and validated through the field testing. Direct feedback from the pilot in the field is very important. The light approaching system is mostly useful at night landing, so the field testing must be carried out at night. There is a large effort to set up the field testing with a local airport because of the complexity of air trafficking. We were lucky to have a volunteer pilot from the college who conducted the flight with the students and provided feedback. The ground crew has to communicate with the sky for different testing purpose. It takes effort and iteration in the process. For example, Figure 1 shows the successful lab testing and the failure of the initial field testing because of the interface issues of airport power system and prototypes. The final field testing performance was shown in the Figure 2 as the light bar is visualized in the sky. Considering the complexity in logistics and safety needs, this was an extremely rare kind of testing that happened in Clinic program.

5. Additional considerations include thermal consideration and cost analysis. The fact that incandescent lights melt ice and snow naturally because of inherent heat. LED lightings are much more power efficient which is the main drive of the project, but it doesn't handle ice and snow on its own. Therefore, an additional heating system and control/sensing system should be considered if such system is deployed at locations weather encounters ice and snow regularly. In additional to the prototype cost, some estimate of the overall system cost was also included.

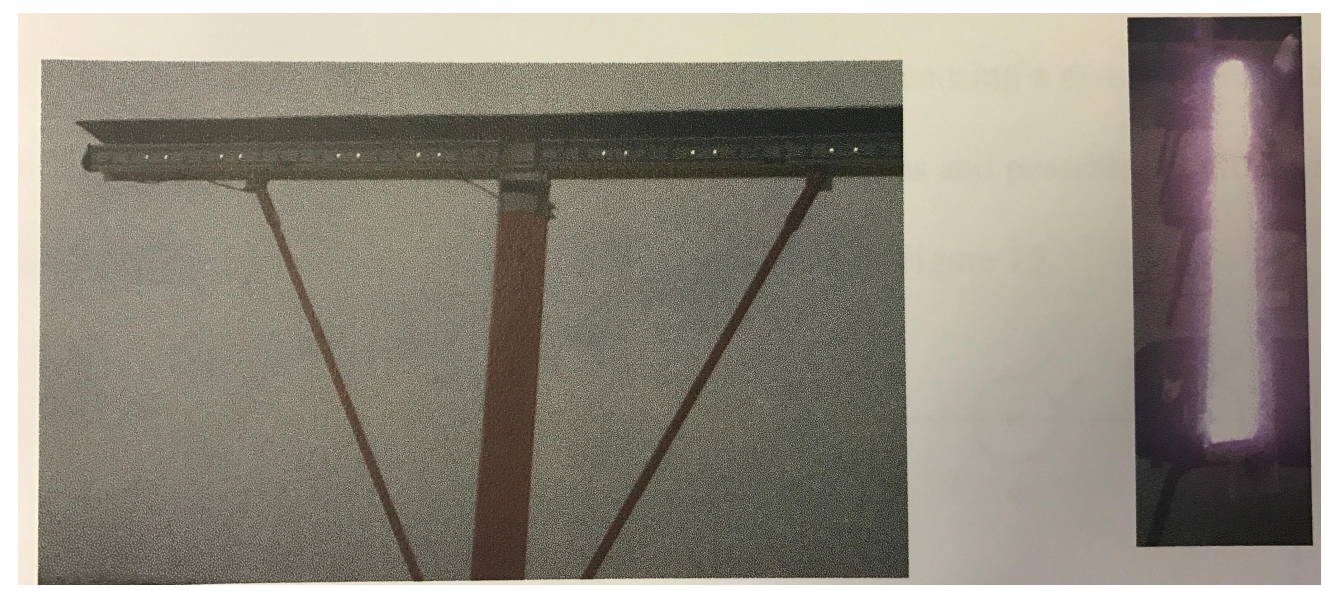

Figure 1(a). Failure in the initial field testing (b) Successful lab testing with brightness measurement 


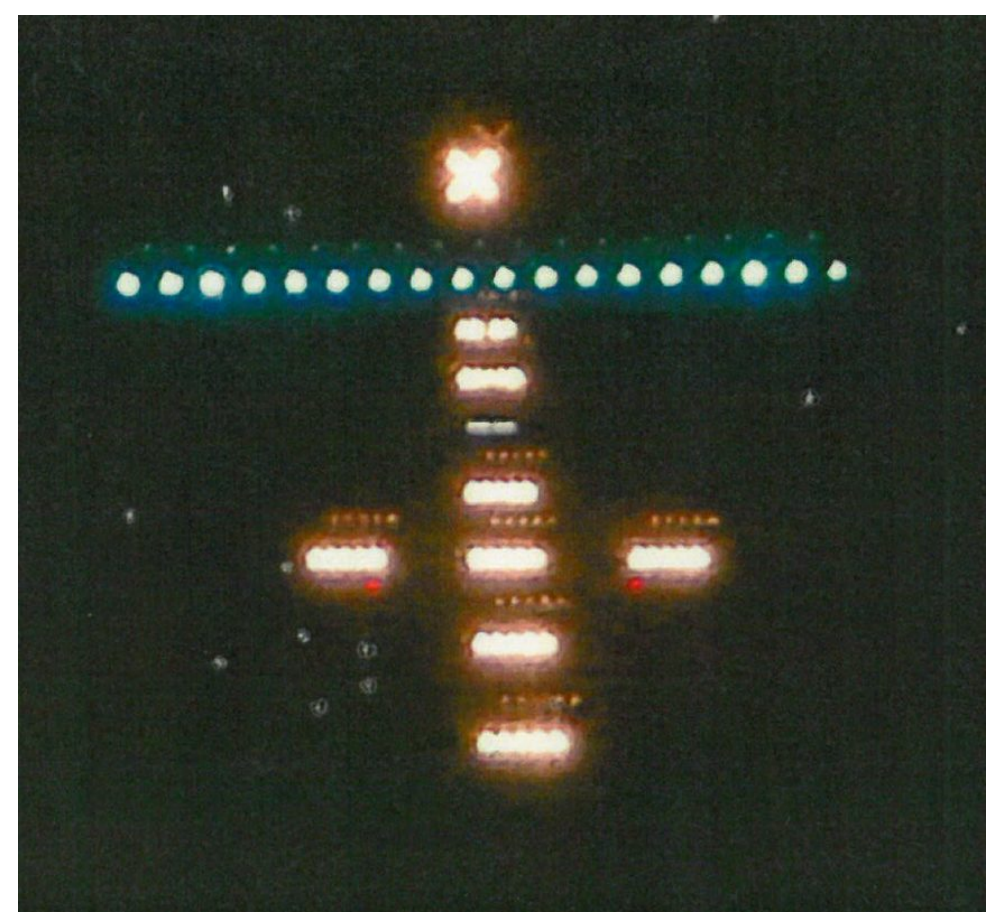

Figure.2 Field testing result of LED light bar for MALSR system in Ontario Airport

Some limitations and further development of the project:

1. At the time, the Luxeon LED were fairly early generations of the devices, and the focusing optics was not quite ready at the time of the prototyping. Therefore, the angle of light distribution is rather large. In the far distance of space, the amount of lights that approaches the aircraft is rather small portion of the overall light intensity from the devices. Therefore, it appears dim and thin strip of lights in comparison to the existing system. More light bulbs and power might be needed to increase the brightness of the light bar and width of the light bar.

2. The following year, a different Clinic team tested new LEDs with better focusing optics. Extra focusing is not good either, because it also has to support the flight range (both height and distance of travel) where the approaching lights need to be visibly brighter. In principle, a more specialized focusing lens that supports or optimizes for such range could be incorporated, but it is not readily from manufactures and easily tested. In addition to the static performance, the follow up team also introduced and explored a potential flashing mode that can easily adjust the flashing pattern for the pilots, and increase the visibility needs of the approaching system. It provided a very promising solution.

\subsection{Project 2: Uniform Illumination for Fluorescent In Vivo Imaging - UVP}

Optical fluorescence in vivo imaging is an important tool to evaluate biological changes in living specimens for tutor biology and drug discovery. UVP is a company focusing on UV sources for optical fluorescence bio-imaging systems. One of the challenges is to have uniform illumination in an enclosure of a small cabinet size, and it is specified as $25 \mathrm{~cm} \times 25 \mathrm{~cm} \times 35 \mathrm{~cm}$ for a typical size of the UV imaging system that UVP carries in their product line. The uniformity is a critical condition in such instruments because otherwise the brightness of biomarker fluorescence will be skewed to reflect the true quantitative measurements. Any variation in light intensity incident on the sample creates artifacts in optical fluorescence imaging data.

The team was a joint team with engineering students from HMC and management and marketing students from Keck Graduate Institute (KGI). The market analysis was important to the UVP for its product developments in that time frame. As a result, the engineering team of 3 students was also less than a typical Clinic team size involved in design and prototyping. The team completed four design alternatives and built three different prototypes. The uniformity of 
illumination, which was the most important performance, was evaluated experimentally as well as in TracePro, a raytracing simulation software. There are a few main challenges students encountered:

1. Design various alternatives that fit to the specified space. The team explored 4 different designs all with the uniformity requirement in mind. The light bulbs must be incorporated mechanically and electrically, and uniformity must be achieved through the clever design. The following three examples illustrate completely different approaches to provide uniformity. In the first design shown in Figure 3(a), everything is static, and the ring of lights in the bottom of the dome in combination of the full reflection coating and dome shape provide the uniformity of lighting in 3-D space. The second design idea is to use 2-D uniform light sources such as fiber optic panels to assisting forming 3-D uniformly lighted space. The design alternative in Figure 4 requires fast rotation of the arc where a set of bulbs are located evenly to provide 3-D uniformity. This design requires mechanical design and machining skills as well as proper electrical set up for the lights and motors. The exact same design is used as the uniformity detection apparatus if the light bulbs are replaced by photodiodes in the strategic positions for volume uniformity (angle, height) measurement.

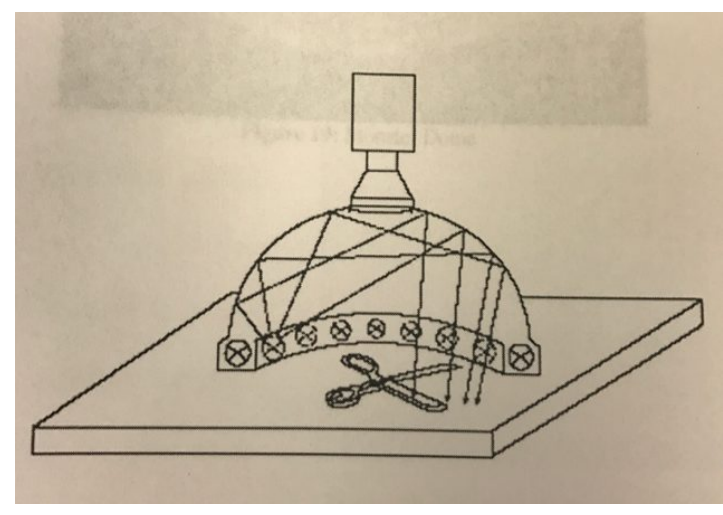

Figure 3. (a) Reflective dome lighting prototype design

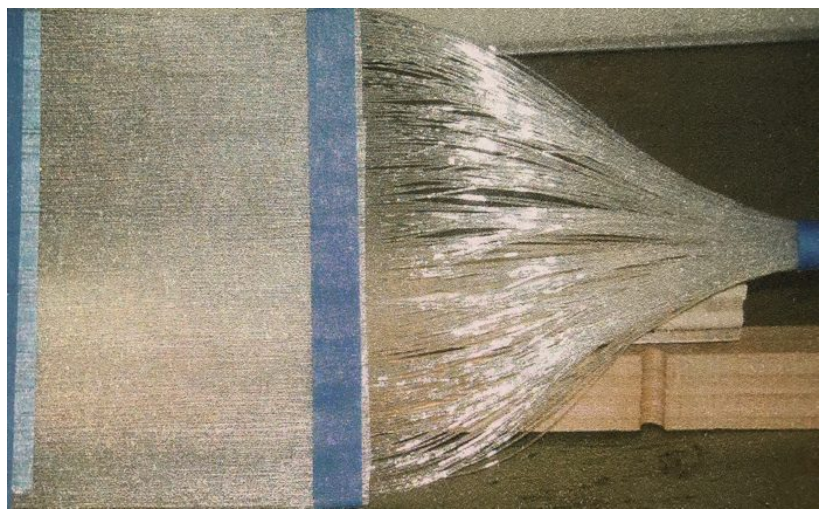

(b) Fiber optic panel for 2-D uniform light source component

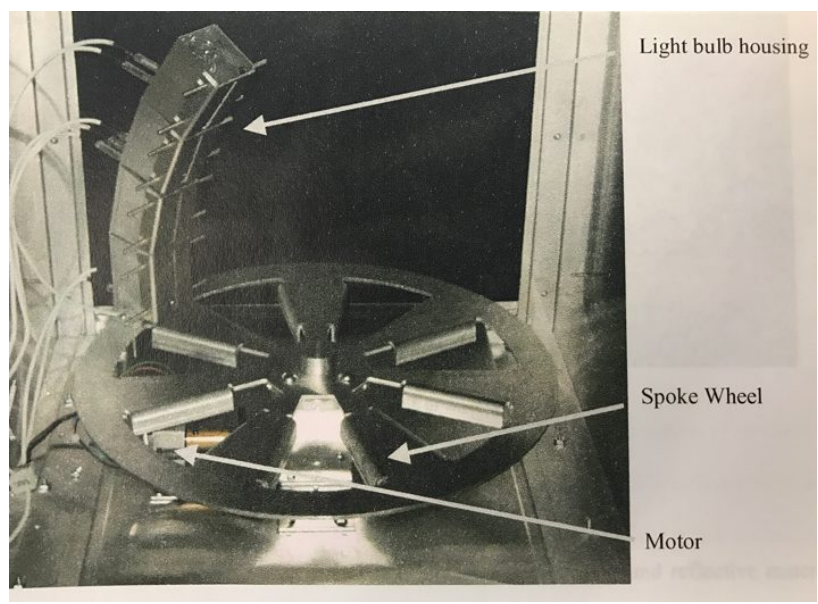

Figure 4. Rotating arc prototype for both light sources as well as uniformity testing apparatus

2 Specify the uniformity metric. Measuring uniformity in 3-D space is not an easy task. Even though there are many existing metrics for different purposes, the team still have to decide on which measure is appropriate and good fit for the application. Statistical quantities Kurtosis (measure distribution tails in a set of data) and coefficient of variance $(\mathrm{CV})$ have been chosen as the 2-D uniformity. A specification of $7 \% \mathrm{CV}$ and -1 of Kurtosis is given by the UVP as a benchmark. Both angular and planar illumination data are evaluated using the statistical analysis to form an understanding of 3-D uniformity. The team has to develop Matlab scrips to automate the reading of the data and statistical analysis of the data. The hemisphere testing devices as in Figure 4 provides two angular degree of freedom, and positions provide increments of 16 degree increment. 
3 Making the prototypes involve a lot of hands-on work. Each design includes some mechanical construction, circuits for light bulbs. For example for the fiber optics panel prototypes, due to the high cost of commercial product, the team create a winding system inhouse, and tried sandblasting to create uniform removal of the cladding layer for lights to uniformly emit from the panel. Chemical etching was also experimented to examine the easier control of the process.

4 Both the experimental testing and simulation were completed to validate the uniformity specifications. For the experimental data, the same arc design shown in Figure 3 was used for a set of photodiodes to collect brightness information in the given coordiate system and 2-D planes. A map of two angular dependance over the whole volume can also be generated. Data acquisition and synchronization with the motor driven system is set up as well as the post-procession of data. In comparison to current light system (current product line) performance of $8.2 \% \mathrm{CV}$ and -0.3 of kurtosis, the rotating arc design performed well with $2.82 \% \mathrm{CV}$ and kurtosis of -0.8 . In addition, Tracepro analyzed and validated the feasibility of the dome design concept. Angular mapping illustrate the spatial symmetry or assymetry, so it provides additional insights of the 3-D spatial uniformity performance.

5 As a part of the business development, the team also pursued SBIR grant submission. This was an excellent opportunity for the team to fomulate the project aims, describe the usefulness of the innovation, provide preliminary data and research findings of the Clinic project, as well as the overall plan of the proposal.

\subsection{Project 3: Redesigning the Lighting and Optics for Laparoscopic Surgery - City of Hope}

This is an on-going Clinic project this year on redesigning the lighting and optics for laparoscopic surgery. Laparoscopic surgeries are commonly done to reduce the trauma of large open abdominal (or chest) surgery, and offers faster recovery for patients. The existing set of tools includes the central visualization and imaging tool that contains a light source and camera system. This system is extremely expensive and tethered with high power electrical sources that is stationed next to the operation table, and has been around for more than 20 years. The motivation of the project is to explore low cost and low power technology alternatives. For example, LEDs are much lower cost but provides high luminance with more development of efficiency, and small cost effective camera systems are readily accessible with the rapid development of the smart phone technology. This is an exciting project to potentially disrupt the technology in surgeries, and provide more opportunities for medical conditions in underdeveloped regions. The team would examine the feasibility of the designs, how the performance of lighting and image quality in comparison to the existing system, and how other features could be incorporated as a result of such a big shift in devices. Because this is an on-going project, I will provide the description in terms of context of the problem and specific challenges. The explored solution and directions are summarized without getting to the specific final details of the design.

There are some interesting challenges in this particular project:

1. The size of the tools is small. Even though we are introducing a completely new technology into the existing surgery, because of the standard ways of conducting these surgeries, the size of the tools that the design must fit in limit the solutions to a rather limited small number of choices. In addition, for safety and manufacturing cost consideration, the designs must be simple and reliable. Figure 5 illustrates typical surgical setting and tool sizes. The trocar sizes are typically range from $5 \mathrm{~mm}-12 \mathrm{~mm}$ in diameter, maybe rarely to $15 \mathrm{~mm}$ to maintain the small incisions for such a surgery. The light for illumination and the camera for images/videos to transmit to outside display monitors should be integrated as much as possible. Multiple light sources in different incision might be desired to create shadow and depth perception which is one of the shortcomings of the single light source setting of the current system. A central trocar that allows for the surgeons to conduct the observation with sufficient lighting is necessary for precision of surgical procedure. The team finds there is tradeoff between the size of the tool design and the space to accommodate brighter lights thus better performance. Adjustable light intensity is also desired for different operation conditions and for power reservation needs. Careful decisions should be made, and variation of the designs should be offered for the liaisons/doctors for their feedback.

2. The team focuses on the mechanical design of the lights that fit with exiting tools, and makes it more versatile. A direct feedback from the liaison who are doctors in this case are extremely valuable, so that teams can decides on the need for the surgical setting. Observation of the actual surgery was arranged to see the typical operation habits of the doctors during such surgeries. These user experiences are critical in the prototype design phase. Similarly, the team conducted several field tests to evaluate the lighting and camera system prototypes in an 
emulated surgical setting. A direct comparison with the existing system is possible. These are all critical steps in the successful outcome of the project.

3. There are some in house manufacturing limitation for the prototyping stage. While 3-D printing is readily and conveniently available for fast prototyping and idea validation, some of the more sophisticated designs require more precise control of the material such as plastics become a limiting factor. For example, a plastic tube with precise control of the inner diameter and outer diameter, or curvature required for different size needs (small for the lower end for trocar size requirement, but larger to accommodate circuits and battery at the bub area). Even though once we have the idea and design, injection-molding could be used to achieve these needs, it was not as accessible to the team and the manufacturing requires longer time and more initial cost, so we have to stay in the proof-of-concept stages. In a few cases, students have to stay with a slightly larger size for the ease of machining.

4. There are many choices for the camera. For the necessary resolution, higher performance cameras are desirable. It is not easy to integrate the camera chip and transmitter/receiver circuit, with the lighting tools for the given space. A more complete design and design iterations need to happen within the time frame. This sometimes pose a challenge with the multiple aspects of requirement (spatial needs, power/intensity requirement, manufacturability, system integration).

5. In this particular case, the team is more hands-on mechanically than electrically, so the final prototypes and deliverables achieves more success in the mechanical design aspects than the electrical implementations. Students background could potentially shift the direction of the development when multiple goals compete with the students' time and contribution. Feedback from the liaisons also influence the direction of the development.

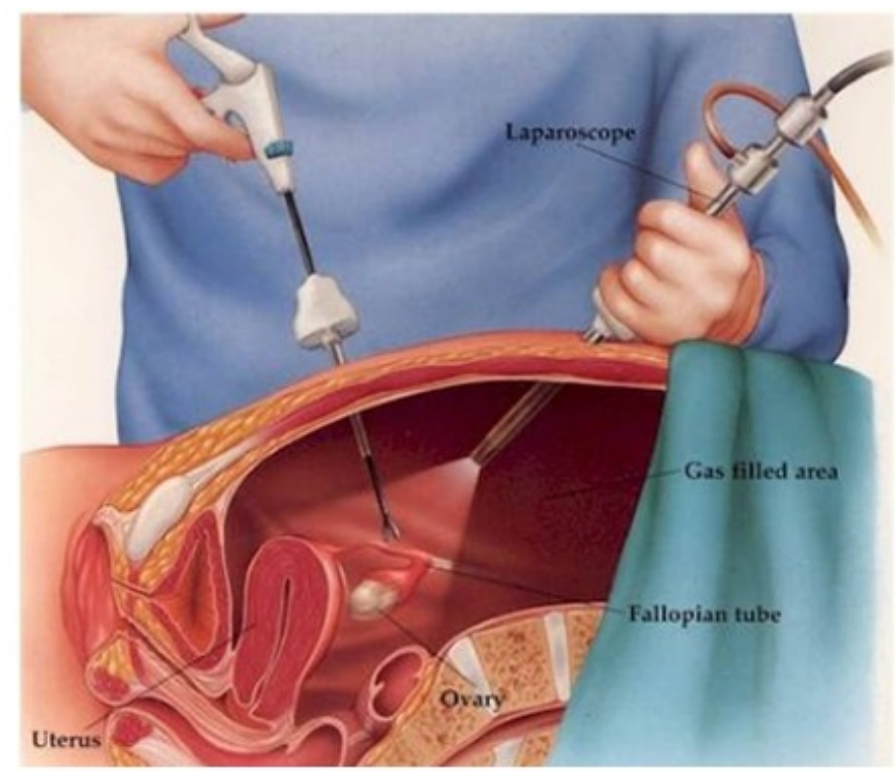

Figure 5. Laparoscopic instruments. [2] 
Table 1: Summary of technology and disciplines involved in the three sample Clinic projects

\begin{tabular}{|c|c|c|c|c|}
\hline Year & Sponsor & Project Title & $\begin{array}{l}\text { Optical Technology } \\
\text { Involved }\end{array}$ & $\begin{array}{l}\text { Other Engineering } \\
\text { Discipline and } \\
\text { Challenges }\end{array}$ \\
\hline $\begin{array}{l}2002- \\
2003\end{array}$ & $\begin{array}{l}\text { Federal Aviation } \\
\text { Administration } \\
\text { (FAA) }\end{array}$ & $\begin{array}{l}\text { Update and Integration of } \\
\text { LED Airport Approach } \\
\text { Lighting }\end{array}$ & LED, lens & $\begin{array}{l}\text { mechanical, electrical } \\
\text { and power design, } \\
\text { field testing, } \\
\text { prototype deployment }\end{array}$ \\
\hline $\begin{array}{l}2005- \\
2006\end{array}$ & $\begin{array}{l}\text { Ultra } \\
\text { Product } \\
\text { (UVP) }\end{array}$ & $\begin{array}{l}\text { Uniform Illumination for } \\
\text { Fluorescent In Vivo } \\
\text { Imaging }\end{array}$ & $\begin{array}{l}\text { UV light sources } \\
\text { Ray tracing } \\
\text { simulation }\end{array}$ & $\begin{array}{l}\text { biomedical imaging, } \\
\text { statistical analysis, } \\
\text { data acquisition, } \\
\text { testing } \\
\text { business/market study }\end{array}$ \\
\hline $\begin{array}{l}2016- \\
2017\end{array}$ & City of Hope & $\begin{array}{l}\text { Redesigning the Lighting } \\
\text { and Optics for } \\
\text { Laparoscopic Surgery }\end{array}$ & $\begin{array}{l}\text { LED, high resolution } \\
\text { camera }\end{array}$ & $\begin{array}{l}\text { mechanical } \\
\text { machining (3D } \\
\text { printing), electrical } \\
\text { circuits, testing in } \\
\text { surgical setting, } \\
\text { system integration }\end{array}$ \\
\hline
\end{tabular}

\section{UNDERGRADUATE LEARNING SKILLS}

These optics projects in Clinic work well for the undergraduate learning skills. The main lesson might be learned is the careful scoping of each of the projects. The well-structured program is highly valuable for supporting the undergraduate projects. It facilitates the schedule management of the whole project, and the expertise of the faculty is mainly from schedule and team management perspective than from technical guidance focus. The support of the liaisons from the industrial sponsor not only provides more direct technical guidance and expectation, but the relationship is a good balance of pressure and mentorship. The faculty usually provides a lot of soft support regarding schedule management and the next immediate task feedback, and it also provides a lot of support for feedback in presentation and written documents. The technical support is intentionally limited to be small so that within the Engineering department, it provides a lot more flexibility of matching projects and faculty specialties, but more importantly creates the culture of student ownership in the final technical solution and ideas. This provides a healthy environment of creativity and freedom given the guidance of time structure and specific deliverable and expectation from the combined academic needs and sponsor needs.

The project level (even for optical projects) should include a broad set of principles so that different students can contribute to different aspects of the project, which you can pull out students' skills more easily in the undergraduate level. Students in HMC are not specifically trained for optical engineering area, so a project too specialized and narrowed in that area would require all the students to pick up learning in the discipline, and put more pressure on the learning curve as well as challenge of team contribution to the same area. A broader based project that centralized on optical devices can provide a good balance between what students' background and what they need to learn on the fly during the project. For example, all of these sample projects involve prototype designs (creativity), mechanical construction, electrical circuitry set up, and maybe modeling and simulation tools for performance evaluation. If other institutions that have an engineering curriculum focusing more heavily on optics training, then they might strike proper balance differently (optics vs. non-optics discipline). For HMC Clinic students, half and half breakdown seems a good starting point.

The hands-on project is a good model to incorporate undergraduate skills such as writing, communication and leadership. These come naturally as the element of project needs. The overall structure support and framework is helpful to provide enough of freedom and wiggle room for mistakes, but avoid complete chaos or disaster in project management. This might be necessary for undergraduates as they lack previous project management skills. Even though there are plenty of team work opportunities in other course works, a yearlong project management and experience can be quite different and 
daunting. Faculty guidance and previous junior Clinic experience and exposure provides good transition from dependent to independent engineers.

\section{CONCLUSION}

In a brief summary, we believe HMC Engineering with strength in the system and design area allows the students to incorporate multiple aspects of a typical optical project such as mechanical design, electrical circuitry design, prototyping design and testing, quantitative analysis and simulation evaluation. A broad range of projects would be good fit for the undergraduate students with sufficient background in electrical, mechanical and design. The core optics part of the project should be kept at a reasonable level for students to pick up from their research easily even if their background is not particularly strong. More specialized program with stronger curriculum support in the optical engineering could potentially consider projects that require deeper analytical and theoretical background in optics. Local sponsors/industrial partners always provide more convenience for technical support and feedback, which is critical to maintaining the strong communication between the students and the sponsor throughout the span of the project. The optical project experience in HMC Clinic indicates the great potential to bringing optical hands-on project in the undergraduate level. A team structure provides flexibility in adjusting the scope of the project and bringing in broader multidisciplinary aspects that's beyond the core optics. The financial model of the Clinic program support might not be easily transferrable to other institutions because of the long-established history, however the scope and how much a team of undergraduates can accomplish in these projects can be used as a good reference model for other programs as they seek and connect with local industry sponsors to their optical educational program. Somewhat surprisingly, industries beyond optical industry are better places to look for opportunities because these traditional industries just start to change and update their infrastructure and ways of operation by incorporating many recent developments from the optical engineering industry.

\section{REFERENCES}

[1] https://www.hmc.edu/engineering/clinic/

[2] J. Yasny and R. Soffer, "A case of a power failure in the operating room," Anesthesia Progress, vol. 52, no. 2, pp. 6569, Jun. 2005. 